\title{
Class Dojo As Indonesian Language Learning Media At Senior High School
}

\author{
Hamdani, Hamdan Hidayat, Agus Mulyanto, Yessy Hermawati \\ \{hamdani@fkip-uninus.ac.id ${ }^{1}$, hamyat@fkip-uninus.ac.id ${ }^{2}$, agus.mulyanto@fkip-uninus.ac.id ${ }^{3}$, \\ yessy.hermawati@fkip-uninus.ac.id ${ }^{4}$ \} \\ Universitas Islam Nusantara, Bandung, Indonesia ${ }^{1,2,3,4}$
}

\begin{abstract}
Some of the problems in Indonesian language learning at senior high schools today are the lack of use of interactive learning media. This is because the Indonesian learning materials project on the mastery of contextual texts. Not a few teachers only use the text in textbooks. As a result, the management of the class becomes less alive, while the demands of the curriculum require student-centered learning. Class Dojo comes as a class management application. By using the Class Dojo as an Indonesian Language learning media,, it is expected to be able to revive the atmosphere of learning in the classroom. The use of the Class Dojo as a learning medium must be accompanied by the optimization of the sub-modalities possessed by students.
\end{abstract}

Keywords: Indonesian Language Learning, Class Dojo, sub-modalities.

\section{Introduction}

One of the main keys to the success of education in Indonesia is the quality of teachers and curriculum as the legal basis for the implementation of learning. As good as the curriculum, if the teacher is unable to apply it properly, then the curriculum objectives are difficult to achieve. Therefore, it is necessary to strengthen in the field about the application of education regulations that require each teacher to have four competencies, (1) pedagogic; (2) professional; (3) personal; and (4) social competencies.

Indonesian Language Learning in high schools currently refers to the 2013 curriculum which focuses on mastering contextual texts. Text that must be mastered by students requires critical thinking from students. This condition gave birth to a policy from the government in the process of implementing classroom learning. The policy in question is the use of a scientific approach with reference to the 5 steps of the scientific approach known as $5 \mathrm{M}$, (1) observing; (2) ask; (3) try; (4) reasoning; and (5) communicating. Students are encouraged to find out from various sources of information, not just being told. For this reason, they are involved in the learning process through observation, questioning, trying, reasoning, and communicating.

In applying this scientific approach, the teacher's focus is how to apply the 5M steps in learning. Some teachers still emphasize the transfer of knowledge (telling), this can be seen from the questions that arise in the class that most of them still emphasize what, not why and how questions. Though what is expected, students not only know what (cognitive domain), but also know why (affective domain), and know how (psychomotor domain) with the 
"empowering" learning process. Not a little in the implementation, learning stimulus to students is neglected, so that good classroom management is needed.

Classroom management is one of the keys to the success of the learning process. The role of classroom management teachers is very important especially in creating an atmosphere of learning. In principle, the teacher holds two tasks at once, teaching and classroom management. The first task, teaching, is intended to help all students achieve learning goals. Whereas the second task, management issues are related to efforts to create and maintain conditions in such a way that the learning process can take place effectively and efficiently in order to achieve learning objectives. A teacher's failure to achieve learning goals is directly proportional to the inability of the teacher to manage the class. For this reason, classroom management is needed to make learning effective, one of which is by using the class dojo as a medium for learning management.

Class Dojo (http://www.classdojo.com/) is a free classroom behavior management application that allows teachers to provide feedback to students about individual and group activities[1]. This program can be operated through a computer or mobile device, making it easy to make classes motivated and focused by providing students with positive or negative feedback in the classroom by giving or reducing points for certain activities. Teachers can customize programs with badges, avatars, and specific activity characteristics for their programs. Because students receive feedback immediately, this application helps maintain student involvement and helps teachers in developing a positive and productive classroom learning environment.

The study of the Class Dojo was conducted by Maclean-Blevins and Muilenburg[2], who used the $\mathrm{AB}$ design to evaluate the effectiveness of the program on increasing on-task and reducing negative learning behavior in third grade. Students are given Class Dojo points to display appropriate assignments or behaviors. In addition, independent observers code 10 appropriate and inappropriate behaviors from students. Classrooms were observed before using the Class Dojo and after three weeks of using the Class Dojo, there was a change in the average frequency of the reported behavior.

Student behavior increases rapidly, and students report enjoying the use of the Class Dojo. However, there are major limitations in this study. Only one class participated in the study, which mainly prevented generalization of results. An operational definition of the 10 dependent variables is not provided, which has the potential to limit internal validity and increase replication difficulties in the future. In addition, interobserver agreements and integrity data are not obtained, also creates potential limitations for internal validity. Finally, research design (design $\mathrm{AB}$ ) does not allow attribution of treatment effects to intervention, nor can it rule out various threats to internal validity.

Recently, Dillon[3] evaluated the effectiveness of the Class Dojo with group contingency procedures, Tootling, to reduce disruptive behavior and increase academic involvement in fifth grade. Tootling is a positive peer reporting intervention consisting of observing peers and recording positive peer behavior on a note card, which is then read by the teacher. To access reinforcement as a group, students must reach a number of Tootles. Dillon[3] modifies Tootling by asking students to record prosocial peer behavior using the Class Dojo and posting tootles on an interactive whiteboard. Applying Tootling with the Class Dojo resulted in a substantial increase in disruptive behavior and academics involved in all three classrooms. 


\section{Method}

This research was conducted in class $\mathrm{X}$ in high school in Bandung. The class consists of 35 students; 32 students participated in this study. The class also contains a student with special needs. To begin research, positive learning activities are discussed in collaborative conversations with Indonesian teachers in the class. The Class Dojo is used only to record positive activities to be strengthened. For 10 minutes at the end of each lesson, class scores are delivered to students. This is done to identify activities that need improvement.

Before introducing the Class Dojo system, two observers recorded the frequency of student activities targeted for 90 minutes. The same observer returns after two weeks to record student activity. The Class Dojo is used to record positive activity during the study period. Each category in the observation instrument is structured to identify changes before and after using the Class Dojo.

\section{Result And Discussion}

The representation system is the way humans represent their experiences[4]. As for the way it works, every time there is an experience that enters through the five senses, the brain will encode the information in a certain form. For example, when receiving information in a visual form, the brain will encode the information in the form of images. When information is received in an auditory form, our brains will encode the information in the form of sounds or words. Whereas when we receive information in the form of feelings, the brain will encode the information in the form of feelings or emotions. This representation system will be influenced by sub-modalities as material inputs.

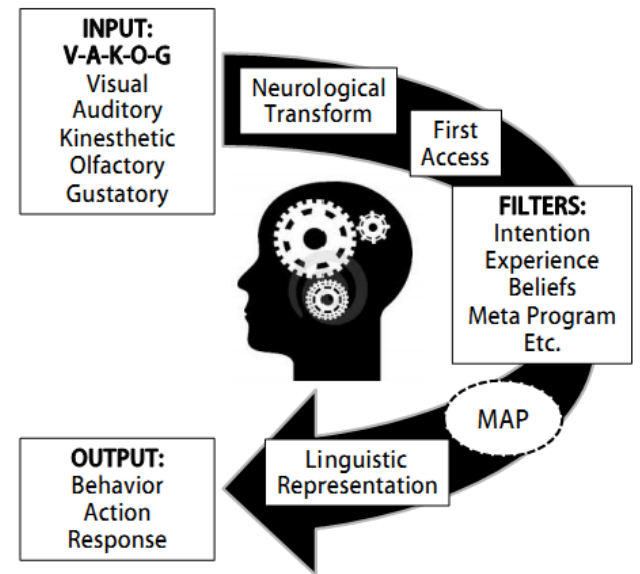

Fig. 1. Representation System

Every student has a sub-modality can be used as initial capital in learning. The submodalities in question are visual, auditory, kinesthetic, olfactory, and gustatory [4]. However, it is very rare for students to have a pure sub-modality. Students who have a more dominant visual sub-modality will optimize their eyes in obtaining information. They are more interested in physical things. Students with visual sub-modalities tend to be high-pitched and 
speak fast, as fast as they visualize their experiences and use body movements. Breathing high and shallow in the chest. They usually like to interrupt other people's conversations and move fast and full of energy. When communicating often use words such as clear, visible, appear, look, show, have a picture, light up, glare, observe, and others.

Types with auditory sub-modalities tend to breathe through the diaphragm. They prefer listening rather than talking. And when speaking they use color variations of broken tones showing as if discussing one picture to another, the tone of the rhythmic medium and tempo. The ability to hear is extraordinary without the penchant for interrupting. The auditory type hears, speaks, and makes decisions based on careful analysis.

Type with kinesthetic sub-modalities, tend to breathe deeply and low in the abdomen. The tone of his voice is slow, a lot of long pauses and sounds deep. They prioritize feelings. Therefore decisions taken are based on feelings and emotions. When communication often uses vocabulary such as touching, feeling, feeling, relaxing, heavy, soft, pinched, and so on. Communicating with the kinesthetic type must try to make them feel what the teacher is saying.

In utilizing the Class Dojo as a learning medium, the teacher first mapped the students' sub-modalities. This mapping is done with a test of suggestibility. This suggestibility test is expected to be able to gather information about students' sub-modalities. The basic principle of the Suggestibility test is to guide students to be able to carry out certain activities through very simple suggestions. The test results will be used as a reference in managing learning through the Class Dojo. The suggestibility test is carried out with the following techniques.

\subsection{Rigid Catalepsy}

Rigid Catalepsy is done by inviting students to order one of their arms to be straight and stiff like steel so that when the command is received by the Subconscious Mind, the subject's arm cannot be completely bent until the student orders it to return to normal[5].

\subsection{Focus Training}

Focus Training is done by inviting students to attach their index finger and thumb so that when the command is received by the Subconscious Mind side, the index finger and thumb are completely inseparable until the student orders it to return to normal. A variation of this technique is to ask students to make their index fingers straight and stiff (like Rigid Catalepsy)[5].

\subsection{Locking The Hands}

Locking The Hands is done by inviting students to order the two palms to lock tightly with each other so that when the command is received by the Subconscious Mind, the two palms of the subject cannot be opened completely until the student orders it to return to normal[5].

\subsection{Eye Catalepsy}

Eye Catalepsy is done by inviting students to instruct their eyes to be tightly locked so that when the command is received by the Sub-Conscious Mind side, the student's eyes cannot really be opened until the student orders them to return to normal[5]. 


\subsection{Relaxation Training}

Relaxation Training is done by inviting students to be able to order themselves to enter total relaxation, so that when the command is received by the Subconscious Mind, the student's body is truly very relaxed, and may not even be able to move until the student orders it to return to normal[5].

After understanding the sub-modalities of the students, a learning design was prepared by utilizing the results of the suggestibility test. Furthermore, the learning design was accommodated into the Class Dojo as a medium for learning Indonesian. Along with the planning of Indonesian language learning, students continue to carry out learning without using the Class Dojo. Before introducing the Class Dojo system, two observers recorded the frequency of student activities targeted for 90 minutes.

In applying the Class Dojo as a medium for learning Indonesian, students are first introduced to the Class Dojo. Students are given an understanding, that during learning using the Class Dojo, their activities will always be recorded in the Class Dojo application. This is done to provide a verbal stimulus to accommodate students' auditory sub-modalities. In the introduction of the Class Dojo, each student gets an avatar as their identity in the Class Dojo. This avatar is expected to be an initial stimulus in accommodating students' visual modality. To accommodate students' kinesthetic sub-modalities, researchers formulate strategies directly in learning

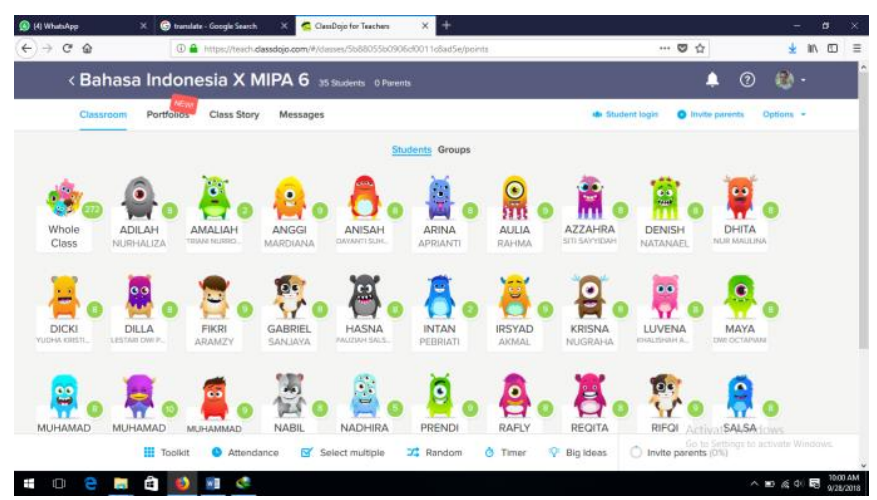

Fig. 2. Class Dojo Start Preview

In each lesson, student activities will always be recorded in the Class Dojo by referring to the observation instrument that has been compiled. The observation instrument includes discipline, hard work, cooperation, helping friends, the ability to answer the right questions, and speaking politeness. Every student who meets the criteria for the instrument will be given points that will accumulate until this research is complete. Achievement of student points will be announced in the final 10 minutes of learning. This is expected to be a stimulus for students at the next meeting. This will also be a reference for researchers in planning their next learning. 


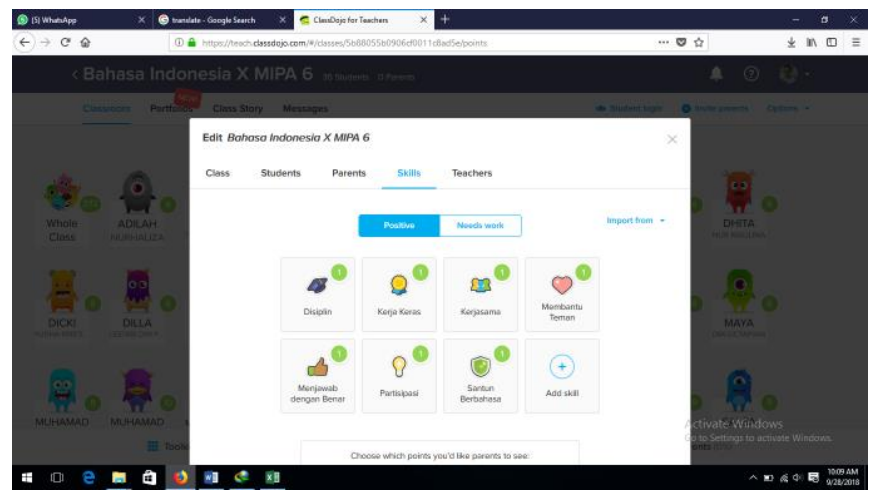

Fig. 3. Observation Instrument for Student Activity

In the early stages of the learning process researchers do 'building rapport' to build good relationships between students and teachers. This is done using the NLP technique. Building rapport is carried out by utilizing the results of the suggestibility test conducted before the planning of learning.

The second step is to do 'pacing' or equate position, gesture, a language with other people or students. In this pacing stage, there are two phases, 'verbal pacing' and 'non-verbal pacing'. Informing a common position through verbal pacing or communication through verbal and written teaching educators ask students questions about their readiness to participate in learning together. In communicating verbally, the word used is a positive word, and in the form of repetition with the aim of affirming the conditions to be faced. The purpose of this pacing is the agreement between students and teachers in the learning process (see transactional psychology). At the stage of relationship formation through "non-verbal pacing" there are several techniques of pacing:

1. Mirroring or mirroring by imitating students' body movements which will bring the teacher closer to students.

2. Eye Contact will increase student confidence.

3. Eye Alignment, in other words, the students' eyesight will show their condition.

4. Verbal agreements focused on non-leading comments will make students freer to express their problems.

5. Breathing is imitating the student's breathing pattern which will bring the teacher closer to the students.

6. Abreaction or emotional release that must be addressed naturally.

The third scheme, 'leading' which means leading or directing after the pacing process we do, after doing a pacing, students will feel comfortable with the teacher. At this stage, there are three techniques, namely

1. Induction

Induction is done by giving speeches to students by utilizing the results of pacing. Induction carried out in the leading process will help to establish the right direction[6].

2. Framing

Framing is done to instill a new concept in students. Framing is used after the teacher knows how the perspective or way of the view of students towards teaching material[6].

3. Re-framing

Sometimes students have misconceptions. Re-framing is done to change the wrong initial concept and replace it with the correct new concept[6]. 
That's when almost everything that the teacher says or assigns to students, students will do it happily. This is because the students' subconscious mind will capture our subject matter is easy, so no matter how difficult the exam questions are tested, it will be easy, and students will be able to achieve brilliant learning achievements.

The fourth stage, namely modeling. Modeling is the process of giving examples or examples through consistent speech and behavior. This is very necessary and becomes one of the keys to learning. After students become comfortable with us. Then it is also necessary to trust students to the teacher with our behavior consistent with our words and teachings. So that we are always a trusted figure.

The next stage, anchoring is an artificial program to generate an instant State of Mind (eg, confidence, calm, etc.). The way to make an anchor is by doing an Elicitation (withdrawal, generation) of a certain State of Mind (which is in accordance with the Anchor that will be formed) from within the student, can be done through Positive Age Regression. Next, amplify, and at the main stage do the association between the anchor and the state of mind. This is a technique that can be used in making an anchoring.

The sixth stage or the last stage is called evaluation. In the evaluation session, there are two parts that are very closely related to each other, namely reward and punishment. Evaluation in the form of praise is one way to shape one's self-concept. Give praise sincerely to students. With praise, someone will be encouraged to do more than before. Whereas Punishment (punishment) is one of the negative mental block forming on students in the form of verbal or action, avoid giving punishment as much as possible.

After two weeks, observers who observe learning before using the Class Dojo will return to the class to record student activities. The Class Dojo is used to record positive activity during the study period. Each category in the observation instrument is structured to identify changes before and after using the Class Dojo. Their observations will be used as a reference for analyzing the development of positive activities among students before using the Class Dojo and after using the Class Dojo as an Indonesian language learning media.

The end result of learning shows that, positive activities of students tend to increase. The biggest increase is the ability to work with students and the hard work of students in carrying out learning. This can be seen in Figure 4. The level of student participation in learning tends to increase. Learning interactions using Class Dojo tend to be more effective, because of the optimization of students' sub-modalities in learning.

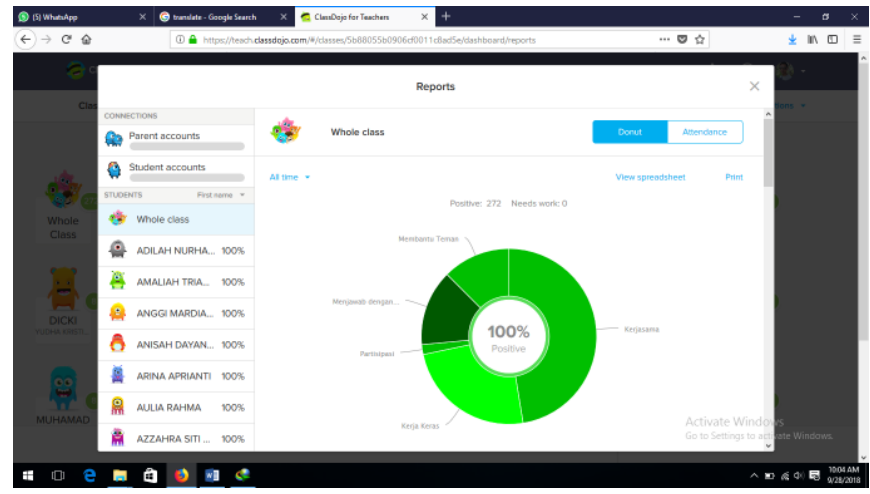

Fig. 4. Diagram of Increasing Student Activity 
To complete the extent to which the Dojo Class role as a medium of Indonesian language learning, data is needed to evaluate the acceptability and the immersion of learning media. This acceptance and feasibility were obtained through questionnaires for students and observers as users of learning services. Thus, acceptance and weakness will be assessed through assessments of parties related to learning. The following is the result of distributing questionnaires distributed to students and teachers.

Table 1. Recapitulation of the Questionnaire, Utilization of Learning Media

\begin{tabular}{|c|c|c|c|c|c|c|}
\hline \multirow{2}{*}{ No. } & \multirow{2}{*}{ Questions } & \multicolumn{5}{|c|}{ Frequency } \\
\hline & & 1 & 2 & 3 & 4 & 5 \\
\hline 1. & Clarity of the media in presenting concepts & - & - & 24 & 2 & 5 \\
\hline 2. & Clarity of the media in presenting procedures & - & - & 23 & 4 & 4 \\
\hline 3. & Clarity of the media in presenting the principles & - & - & 24 & 4 & 3 \\
\hline 4. & Suitability of media with learning objectives & - & - & - & 19 & 12 \\
\hline 5. & Media suitability with your level of ability & - & - & - & 26 & 5 \\
\hline 6. & Systematic presentation of material & - & - & 21 & 2 & 8 \\
\hline 7. & Presentation of relevant examples & - & - & - & 11 & 20 \\
\hline 8. & The attractiveness generated by the media & - & - & - & 18 & 13 \\
\hline 9. & Clarity of the letters displayed & - & - & 10 & 21 & - \\
\hline 10. & The color harmony displayed & - & - & 16 & 15 & - \\
\hline 11. & $\begin{array}{l}\text { Appropriate display of images as illustrations relevant } \\
\text { to the material }\end{array}$ & - & - & - & 28 & 3 \\
\hline 12. & $\begin{array}{l}\text { Suitability of graphic display to illustrate charts or } \\
\text { percentages (if any) }\end{array}$ & - & - & - & - & - \\
\hline 13. & $\begin{array}{l}\text { Appropriate display of animation variations (motion, } \\
\text { illustration, etc.) }\end{array}$ & - & - & - & 23 & 8 \\
\hline 14. & Sound quality (audio) as backsound supporting display & - & - & 2 & 17 & 12 \\
\hline 15. & Your perception of the use of media used & - & - & - & 24 & 7 \\
\hline 16. & The role of this media in your learning motivation & - & - & - & 23 & 8 \\
\hline 17. & $\begin{array}{l}\text { The role of the media to clarify the understanding of } \\
\text { the material }\end{array}$ & - & - & - & 6 & 25 \\
\hline
\end{tabular}

The questionnaire distributed to respondents basically aims to get an overview of the learning media that has been designed. The direction of the questionnaire questions distributed includes 3 things, namely (1) the appearance of the media; (2) media functions; and (3) the role of the media. The assessment of these respondents will be the basis for further analysis of the needs of electronic learning media as a development effort.

From the table above we can see that the Dojo Class that has been tested is enough to fulfill expectations as a learning medium. However, there are still weaknesses in several aspects. The following is an explanation of the results of the questionnaire and needs analysis in the development of learning media.

\section{Media Display}

The appearance of media is one of the attractions that is one of the focuses in the development of this learning media. A good media display will attract attention to using the media. From this aspect, a number of things will be measured which are the subject of the assessment of media display. The aspects in question are (1) clarity of letters; (2) color harmony; (3) suitability of display with material; (4) the suitability of the graph display to illustrate the chart or percentage (if any); (5) suitability of the appearance of animation 
variations (motion, illustration, etc.); and (6) sound quality (audio) as a backsound supporting display[7], [8].

\section{Media function}

Media function is one aspect that is one of the focuses in the development of electronic learning media. A good media function will attract attention to using the media. From this aspect, a number of things will be measured which are the main points for evaluating the functioning of electronic learning media prototypes. The aspects in question are (1) clarity in presenting the concept; (2) clarity in presenting procedures; (3) clarity in presenting principles; (4) suitability with learning objectives; (5) suitability with ability level; (6) systematic presentation of material; and (7) the presentation of relevant examples [7], [8].

\section{Media role}

The role of media is one aspect that is one of the focuses in the development of electronic learning media. A good media role will attract attention to using the media. From this aspect, a number of things will be measured which are the main points for evaluating the role of electronic learning media. The aspects in question are (1) the role of learning motivation; (2) role in clarifying material; and (3) strengthen perception[7], [8].

\section{Conclusion}

Based on the results of the research above, it can be seen that the activity of students in class X MIPA 6, SMAN 17 Bandung has increased. Theoretically, this increase in activity is due to the stimulus given during the Indonesian language learning process. Stimulus is given by paying attention to the sub-modality of students in learning, visual, auditory, and kinesthetic.

It is important to remember that technology is only a tool. They are not goals but a means to help teachers manage the class better. In addition, the gateway tool presented here is only a representative in a substantial and growing collection of online offers. As stated in the introduction, we believe that for change to occur in the classroom, a paradigm shift must begin with the teacher rather than the student. When teachers see the value of technology and its impact on their classroom practices, that awareness opens the gates for further technological integration.

For teachers, using tools that streamline professional tasks, as mentioned above, can act as a springboard for the use of further technology for classroom learning. Integrating tools such as those mentioned in this article and participating in sustainable, formal or informal professional development, will help build teacher confidence, will demonstrate the value of technology in the classroom, and will increase the use of technology by teachers. Such integration can help set the stage for students to benefit from the instructions embedded in technology into the future.

\section{References}

[1] K. Dadakhodjaeva, "The good behavior game: Effects on and maintenance of behavior in middle-school classrooms using class dojo," 2017.

[2] A. Maclean-Blevins and L. Muilenburg, "Using Class Dojo to Support Student Self-Regulation," in EdMedia: World Conference on Educational Media and Technology, 2013, pp. 1684-1689. 
[3] M. M. Dillon, "The tootling intervention with ClassDojo: Effects on classwide disruptive behavior and academic engagement in an upper elementary school setting (Doctoral dissertation)," The University of Southern Mississippi, 2016.

[4] M. A. Gaffar, A. Mudrikah, Hamdani, and L. L. Hakim, Alphawave Model. Bandung: FKIP Uninus, 2017.

[5] Y. Nurindra, Hypnosis for Dummies, no. November. Jakarta: IBH, 2008.

[6] R. Bandler and J. Grinder, Reframing: Neuro-Linguistic Programming and the transformation of meaning. Utah: Real People Press, 1982.

[7] R. Heinich, M. Molenda, J. D. Russell, and S. . Smaldino, Instructional media and technology for learning, 7th edition. New Jersey: Prentice Hall, Inc, 2002.

[8] J. T. Holden and P. J. Westfall, An Instructional Media Selection Guide for Distance LearningImplications for Blended Learning featuring an Introduction to Virtual World. United States of America: USDLA, 2009. 